\title{
Potential Vaccine Targets for COVID-19 and Phylogenetic Analysis Based on the Nucleocapsid Phosphoprotein of Indonesian SARS-CoV-2 Isolates
}

\author{
Muhammad Aldino Hafidzhah ${ }^{1}$, Renadya Maulani Wijaya ${ }^{1}$, Rasyadan Taufiq Probojati ${ }^{2}$, Viol Dhea \\ Kharisma $^{2}$, Arif Nur Muhammad Ansori ${ }^{3}$, Arli Aditya Parikesit ${ }^{1^{*}}$
}

1. Department of Bioinformatics, School of Life Sciences, Indonesia International Institute for Life Sciences, East Jakarta, Indonesia.

2. Computational Virology and Complexity Sciences Research Unit, Division of Molecular Biology and Genetics, Generasi Biologi Indonesia Foundation, Gresik, Indonesia

3. Doctoral Program in Veterinary Science, Faculty of Veterinary Medicine, Universitas Airlangga, Surabaya, Indonesia

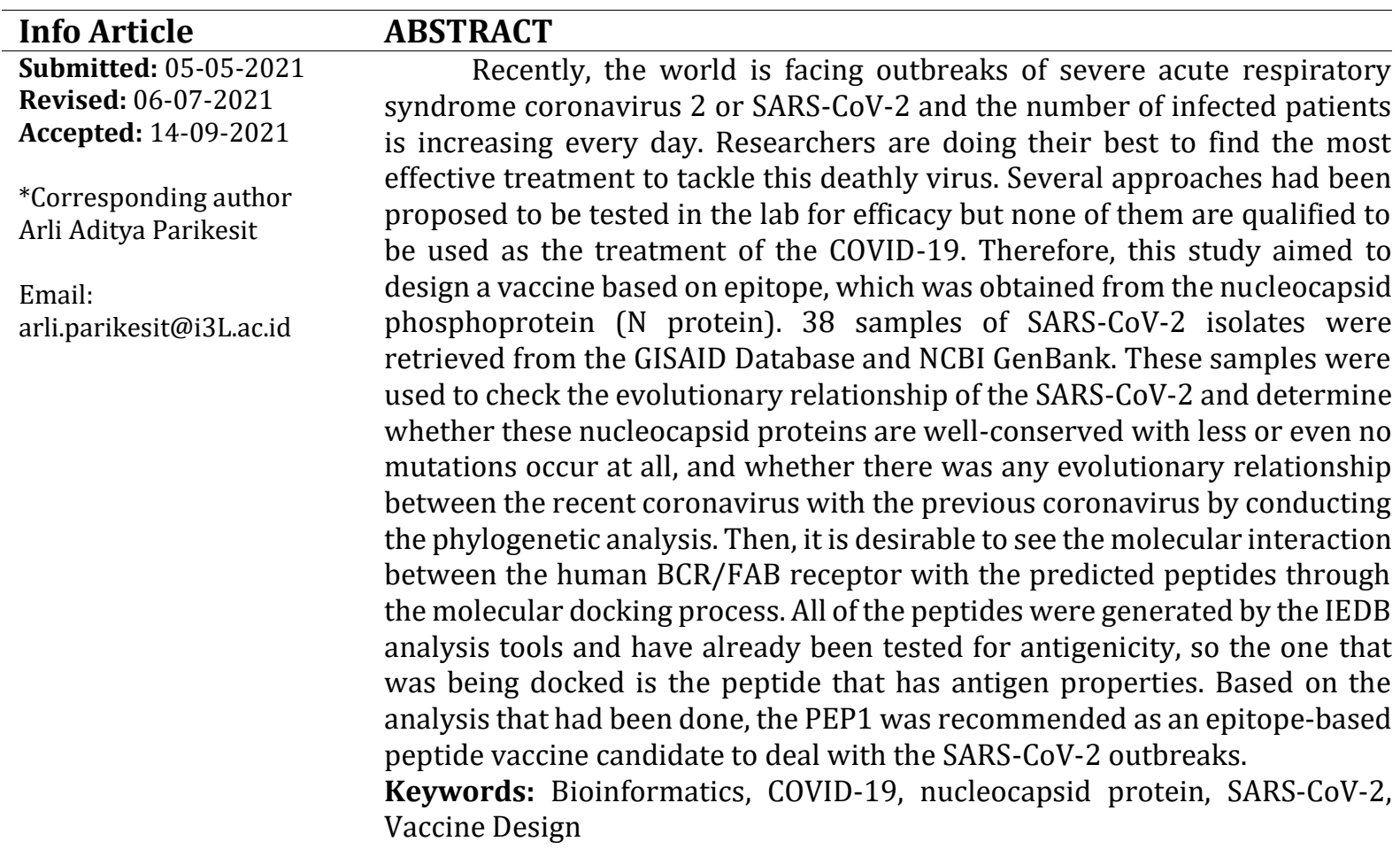

\section{INTRODUCTION}

COVID-19 was known as the disease that was caused by the newly emerging pandemic of Severe Acute Respiratory Syndrome Coronavirus 2 (SARS-CoV-2), which originally came from the Coronaviridae family (WHO, 2020). The number of patients infected with COVID-19 continues to increase significantly every day since the first outbreak in December 2019 in Wuhan, China. According to the data from World Health Organization (WHO), since the first outbreak in every country, the number of confirmed cases had reached 226,000,000 cases worldwide per September 2021. Meanwhile, in Indonesia, the number of confirmed cases has reached 4.190 .763 cases and 140,468 deaths, also per September 2021 (WHO, 2021; Dong et al., 2020). This condition is quite threatening for all the elements of society. Researchers around the world are racking up their brains to develop a way to solve this pandemic and there were tons of approaches had been proposed. One of the methods that had been proposed and might show a promising result is by utilizing the immunoinformatic approaches. Immunoinformatic could be very effective to tackle this problem because these methods utilized the study of immunology, which is about the immune system, and bioinformatics as the methods to determine 
potential candidate compounds for COVID-19 vaccines.

Before going further deep into the vaccine study, basic understandings of bioinformatics, structural bioinformatics, immunology, and immunoinformatic are needed. Bioinformatics is defined as an interdisciplinary study that combines biology, computer science, and statistics to develops methodology and software tools to understand complex biological data available in this world (Lesk, 2019). This study is focused on using some of the bioinformatics "subcomponents" which are structural bioinformatics and Immuno-Informatics. In the field of bioinformatics, there is an area of study, which focuses on the structure, and molecular interactions of a certain biological molecule. Since their main focus is the structure and molecular interactions, this area of study is commonly being used in drug discovery and drug designing. This field of study is known as structural bioinformatics (Brown and Tastan, 2017), and this area of study will leverage the most common tools/technique used for result validation, which is the molecular docking and dynamics.

The structural bioinformatics will be accompanied by another type of study, which combines the study of immunology and the study of informatics/bioinformatics, which are known as immunoinformatic. In scientific definition, immunoinformatic was defined as the interdisciplinary study between experimental immunology and computational approaches (bioinformatics) to help in defining new theories related to immune responses (Tomar and De, 2014). Immunology is a wide field of study, since our study is about designing an epitope-based vaccine, further understanding about epitopes and epitope-based peptide vaccines needs to be obtained. Epitopes were defined as the short and specific amino acid sequences in an antigen that are well recognized by the immune response (Kao and Hodges, 2009). Epitopes are further differentiated into three types, which are the B-cell epitopes, Thelper epitopes, and CTL epitopes. T-helper epitopes are defined as the epitopes that are available on the surface of antigen-presenting cells, which later on will bind with the MHC molecules (Steers et al., 2014). Next is one of the antigen parts, which was shown to be binding with the antibodies, which are called the B-cell epitope (SanchezTrincado et al., 2017).

Those three epitopes are related to each other especially the T-helper and B-cell because if
T-helper cells do not start or trigger any immune mechanism then B-Cell would not be produced and there will be no immune response inside our body, which makes our body prone to be attacked by diseases. For this study our focus is towards the Bcell epitope since this epitope produces an immune response inside our body, and if the virus can be "introduced" with the immune system first by developing a vaccine that utilizes an epitope-based component. Hopefully, when the COVID-19 tries to attack our immune system, our immune system has "prepared" itself. Now, let us know more about the epitope-based peptide vaccine.

Epitope-based peptide vaccine was defined as a type of vaccine that utilizes the chunks of sequences that were taken from antigenic proteins of targeted pathogens which has high immunogenicity (TopuzoĞullari et al., 2020). Epitope-based peptide vaccines are used to overcome the problems that commonly appear on the other type of vaccines. Epitope-based peptide vaccine can overcome safety concerns. The vaccine provides us with a maximal therapeutic efficacy, it is cost and time-effective, it limits the allergenic or reactogenic complications, and also it can be further modified to obtain multi-epitope or conjugated structures (TopuzoĞullari et al., 2020).

That is why in this study, a new methodology was developed in designing a vaccine for viral diseases especially the COVID-19. The methodology that was used in this study was adapted from a methodology that has been developed previously by Kharisma et al., Chen et al., and Bhattacharya et al. in their study about designing an epitope-based peptide vaccine against SARS-CoV-2 with some changes in the parameter and samples that were being used (Muttaqin and Ansori, 2020; Bhattacharya et al., 2020; Chen et al., 2020; Kharisma and Ansori, 2020). This methodology mainly utilized the Immunoinformatic approaches along with bioinformatics software to help in designing and predicting specific epitope-based peptide vaccine, which targets the SARS-CoV-2.

\section{MATERIAL AND METHODS}

The main tools that were used in this research are software and databases that are freely available and accessible online. In this study, the GISAID EpiCoV database and NCBI GenBank were used to retrieved samples or isolates of the SARS-CoV-2 and previous coronavirus outbreaks. GISAID and NCBI GenBank are freely accessible online databases that store sequences related to living 
organisms. In terms of the analysis, prediction, validation, and visualization, online analysis and prediction tools that were utilized in this study are the IEDB Analysis Tools and VaxiJen v2.0 which was used to help in conducting the B-Cell Epitope predictions (IEDB Analysis Tools) and by utilizing the results from the Epitope Prediction to conduct the Antigenicity test with the help of VaxiJen v2.0. These predictions need to be tested for their validity and to do those, molecular docking needs to be done. To support this molecular docking process, CLUSPRO 2.0 Web-based Docking tools were used. To help in visualization, PyMOL 2.4.0 Visualization software was used in this study. The whole process in this study had been summarized in form of a flowchart (Figure 1).

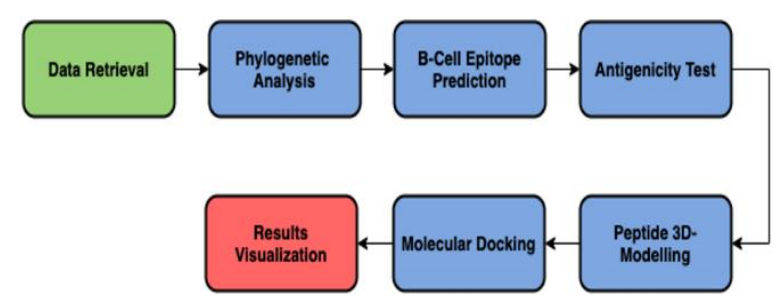

Figure 1. The brief summary of the analysis that had been conducted starting from the sample retrieval to the visualization of the molecular docking results.

\section{Data Retrieval}

The process starts by retrieving samples or isolates from the Global Initiative on Sharing All Influenza Data (GISAID) EpiCoV database and also from NCBI GenBank. In total, 38 Nucleocapsid proteins of the SARS-CoV-2 isolates were successfully being retrieved from both the GISAID EpiCoV database and NCBI GenBank. Nucleocapsid protein was chosen because of its sequence quality. It was shown that nucleocapsid protein has a very well conserved sequence compared to the other proteins available inside the coronavirus structures (Thomas and Gorelick, 2008; Mcbride et al., 2014; Samrat et al., 2020). Global Initiative on Sharing Avian Influenza Data (GISAID) is a global initiative and resource for genomic data of influenza-related viruses (Shu and McCauley, 2017). To support GISAID in terms of the availability of sequences that came from the previous outbreak of coronavirus, NCBI GenBank was used. National Centre of Biotechnology GenBank (NCBI GenBank) is a comprehensive genetic sequence database that provides various types of genetic sequences that came from various types of organisms, including viruses (Benson et al.,
2010). In this process, most of the hCoV-19 samples were obtained from the GISAID EpiCoV database and as stated before, the sample from the previous coronavirus outbreak was taken from the NCBI GenBank since most of the samples from the previous coronavirus outbreak are not available in the GISAID EpiCoV database.

\section{Phylogenetic analysis}

The next process is to conduct a phylogenetic analysis by utilizing the isolates that had been retrieved from the databases (GISAID and NCBI GenBank). The maximum-likelihood tree was generated with the help of MEGA X tools, which is known as the software used for conducting Multiple-Sequence Alignment. The alignment results were then used to generate an accurate phylogenetic tree (Hall, 2013). The purpose of conducting this analysis is to address some issues related to viral research such as the evolutionary relationships and epidemiology of a certain virus (Ansori et al., 2020). In this study would like to address the genetic relationship or evolutionary relationships between the coronaviruses with the help of a phylogenetic tree. In terms of the parameter that are being used, the phylogenetic tree was generated by using a maximum-likelihood method, the Neighbor-Joining algorithms, and the Tamura-Nei model. In terms of the bootstrap method, using bootstrap around 1000 iteration iterated the tree.

\section{B-cell epitope predictions}

Moving on to the next process, which is the epitope prediction using IEDB Analysis tools. IEDB B-cell epitope prediction (IEDB BepiPred-2.0) was used to help in predicting epitopes that might be available in the samples of SARS-CoV-2 isolates with an accuracy of 75\% (Jespersen et al., 2017). In this process, a default parameter was used which consist of the default threshold (threshold score = 0.5). From this prediction, eleven predicted peptides were acquired and those peptides were further tested for their antigenicity using the VaxiJen v2.0 tools.

\section{Antigenicity test}

After the predicted peptides were obtained, the peptides underwent an antigenicity test using the VaxiJen v2.0 online tools. VaxiJen v2.0 is an online web-based tool, which is used to determine the characteristics of immunogenicity or protective antigens (Doytchinova and Flower, 2007). From this test, only 6 peptides were considered as a protective antigen and qualified to move to the 
next step which was the peptide 3D modeling. Meanwhile, the other five peptides were excluded for the next process since they did not have any antigen features, which were needed to develop an epitope-based peptide vaccine. Mostly default parameter was used in this process, only the 'target organism' menu was changed (originally the 'target organism' was in the 'bacteria' option).

\section{Peptide 3D-modelling}

In this process, the six peptides were modeled into 3D structures and the reason why those peptides need to model beforehand is that to conduct molecular docking, 3D structures of the peptides were needed. So, from this process, the 3D-Structure of the six qualified peptides was acquired and these 3D-Structures will be used for Peptide-Peptide docking. In this process, there was no specific parameter that needs to be chosen, so the assumption is that a default parameter provided by the tools was being used. In modeling the peptides, the structure prediction tool used was the PEPFOLD 3 which is a de novo approach aimed at predicting peptide structures from amino acid sequences (Shen et al., 2014).

\section{Molecular docking}

Now, to further validate and determine which peptides will be chosen as an epitope-based peptide vaccine candidate to deal with the SARSCoV-2 outbreaks, molecular docking needs to be done. In this step, the peptides were docked along with the $\mathrm{BCR} / \mathrm{FAB}$ receptor, and their binding scores were measured. A peptide with the most negative binding score was proposed as the epitope-based peptide vaccine candidate to deal with the SARS-CoV-2 disease. In this molecular docking process, the default parameter was used since there were no specific parameters provided by the docking tools. To conduct this molecular docking process, the CLUSPRO 2.0 docking tool was deployed. CLUSPRO 2.0 is known as a web-based tool for the protein-protein docking process, and it provides the user with a simple user interface for basic use. Also, it only requires two main files in the PDB format (Kozakov et al., 2017).

\section{RESULTS AND DISCUSSION \\ Data and isolate retrieval}

In this process, most of the $\mathrm{hCoV}-19$ samples were obtained from the GISAID EpiCoV database and the sample from the previous coronavirus outbreak was taken from the NCBI GenBank since most of the samples were from the previous coronavirus outbreak are not available in the GISAID EpiCoV database. In total, 38 samples were acquired from those two databases (Table I) (appendix).

\section{Phylogenetic analysis}

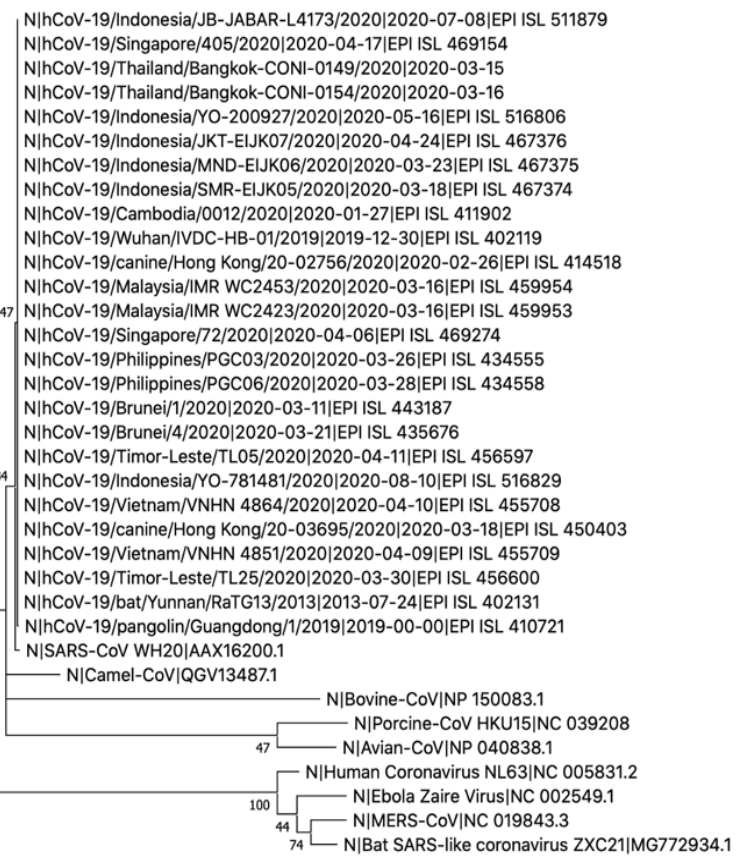

Figure 2. Phylogenetic tree generated to show the evolutionary relationship between the SARS-CoV-2 virus with the other types of coronaviruses that had been emerged in the past decades. the MEGA X software was utilized to generate the maximumlikelihood phylogenetic tree.

The phylogenetic tree (Figure 2) is actual proof that the SARS-CoV-2 still has a very close evolutionary relationship with the previous SARS Coronavirus. It is revealed that there were no significant differences shown in the virus isolates from various countries including those from the Southeast Asia region especially Indonesia. This statement can be proven by seeing the position of the isolates that were in the same clade, which means that they were the same. It does not show very significant differences in terms of their sequences and variants. This phylogenetic tree also shows us that the nucleocapsid protein of the SARSCoV-2 isolates was very well conserved by seeing the result from the multiple-sequence alignment process right before the phylogenetic tree generation process occurred. 
Table I. Samples used for this study. All of the samples were retrieved from GISAID EpiCoV Database and NCBI GenBank. Our study samples were specified to Southeast Asia regions only and some samples from previous coronavirus outbreaks. So, in total there are 38 samples of the coronavirus that will be used in this study.

\begin{tabular}{|c|c|c|c|}
\hline No. & ACCESSION ID & SAMPLE NAME & $\begin{array}{l}\text { SOURCES / } \\
\text { DATABASE }\end{array}$ \\
\hline 1. & EPI_ISL_435676 & hCoV-19/Brunei/2020 & GISAID EpiCoV \\
\hline 2. & EPI_ISL_456597 & hCoV-19/Timor-Leste/2020 & GISAID EpiCoV \\
\hline 3. & EPI_ISL_443187 & hCoV-19/Brunei/2020 & GISAID EpiCoV \\
\hline 4. & EPI_ISL_434558 & hCoV-19/Philippines/2020 & GISAID EpiCoV \\
\hline 5. & EPI_ISL_434555 & hCoV-19/Philippines/2020 & GISAID EpiCoV \\
\hline 6. & EPI_ISL_469274 & hCoV-19/Singapore/2020 & GISAID EpiCoV \\
\hline 7. & EPI_ISL_459953 & hCoV-19/Malaysia/2020 & GISAID EpiCoV \\
\hline 8. & EPI_ISL_459954 & hCoV-19/Malaysia/2020 & GISAID EpiCoV \\
\hline 9. & EPI_ISL_450403 & hCoV-19/Hongkong/2020 & GISAID EpiCoV \\
\hline 10. & EPI_ISL_455708 & hCoV-19/Vietnam/2020 & GISAID EpiCoV \\
\hline 11. & EPI_ISL_455709 & hCoV-19/Vietnam/2020 & GISAID EpiCoV \\
\hline 12. & EPI_ISL_516829 & hCoV-19/Indonesia/2020 & GISAID EpiCoV \\
\hline 13. & EPI_ISL_414518 & hCoV-19/Hongkong/2020 & GISAID EpiCoV \\
\hline 14. & EPI_ISL_402119 & hCoV-19/Wuhan/2020 & GISAID EpiCoV \\
\hline 15. & EPI_ISL_411902 & hCoV-19/Cambodia/2020 & GISAID EpiCoV \\
\hline 16. & EPI_ISL_467374 & hCoV-19/Indonesia/2020 & GISAID EpiCoV \\
\hline 17. & EPI_ISL_467375 & hCoV-19/Indonesia/2020 & GISAID EpiCoV \\
\hline 18. & EPI_ISL_516806 & hCoV-19/Indonesia/2020 & GISAID EpiCoV \\
\hline 19. & EPI_ISL_511879 & hCoV-19/Indonesia/2020 & GISAID EpiCoV \\
\hline 20. & EPI_ISL_469154 & hCoV-19/Singapore/2020 & GISAID EpiCoV \\
\hline 21. & EPI_ISL_467376 & hCoV-19/Indonesia/2020 & GISAID EpiCoV \\
\hline 22. & EPI_ISL_456600 & hCoV-19/Timor-Leste/2020 & GISAID EpiCoV \\
\hline 23. & EPI_ISL_402131 & hCoV-19/Yunnan/2020 & GISAID EpiCoV \\
\hline 24. & EPI_ISL_410721 & hCoV-19/Guangdong/2020 & GISAID EpiCoV \\
\hline 25. & AAX16200.1 & SARS-CoV WH20 & NCBI GenBank \\
\hline 26. & NP150083.1 & Bovine-CoV & NCBI GenBank \\
\hline 27. & QGV13487.1 & Camel-CoV & NCBI GenBank \\
\hline 28. & NC039208 & Porcine-CoV & NCBI GenBank \\
\hline 29. & NP040838.1 & Avian-CoV & NCBI GenBank \\
\hline 30. & NC043505 & Yellow Head Virus & NCBI GenBank \\
\hline 31. & NP579881.1 & Human Immunodeficiency Virus Type-1 (HIV-1) & NCBI GenBank \\
\hline 32. & NC016991 & White-Eye Coronavirus HKU16 & NCBI GenBank \\
\hline 33. & NC019843.3 & MERS-CoV & NCBI GenBank \\
\hline 34. & MG772934.1 & Bat SARS-like-CoV & NCBI GenBank \\
\hline 35. & NC005831.2 & Human-CoV NL63 & NCBI GenBank \\
\hline 36. & NC002549 & Ebola Zaire Virus & NCBI GenBank \\
\hline 37. & EPI_ISL_455947 & hCoV-19/Thailand/2020 & GISAID EpiCoV \\
\hline 38 & EPI_ISL_455943 & hCoV-19/Thailand/2020 & GISAID EpiCoV \\
\hline
\end{tabular}

\section{B-Cell epitope prediction and antigenicity test}

The input for this B-cell epitope prediction is an $\mathrm{N}$ protein sequence which was retrieved from an Indonesian sample (N/hCoV19/Indonesia/JKT-EIJK07/2020|EPI_ISL_467376). This sample was chosen based on the result from conducting a multiple-sequence alignment between five Indonesian samples, which consist of samples from West Java (1 sample), DKI Jakarta (1 sample), South-Sulawesi (1 sample), Central Java (2 samples). Based on the result of the alignment, the sequence of those 5 samples does 
Table IIa. List of peptides generated from the B-cell epitope prediction using IEDB analysis tools, and their antigenicity prediction results which have been calculated by using the VaxiJen v2.0. Peptides that have antigen scores above the threshold proceeded to the molecular docking process.

\begin{tabular}{ccc}
\hline Peptide & $\begin{array}{c}\text { Antigen } \\
\text { (Y/N) }\end{array}$ & $\begin{array}{c}\text { Antigen Score } \\
\text { (Threshold = 0.4) }\end{array}$ \\
\hline NGPQNQRNAPRI & $\mathrm{N}$ & 0.1648 \\
FGGPSDSTGSNQNGERSGARSKQRRPQGLPNN & $\mathrm{N}$ & 0.2916 \\
AGLPYGANK & $\mathrm{Y}$ & 0.5773 \\
HGKEDLKFPRGQGVPINTNSSPDDIGYYRRATRRIRGGDGKMKDLS & $\mathrm{N}$ & 0.2631 \\
GALNTPKDHIGTRNPANNAAIVLQLPQ & $\mathrm{N}$ & -0.1089 \\
TTLPKGFYAEGSRGGSQASSRSSSRSRNSSRNSTPGSSRGTSPARMAGNGGD & $\mathrm{Y}$ & 0.5206 \\
RLNQLESKMSGKGQQQQGQTVTKKSAAEASKKPRQKRTATKA & $\mathrm{Y}$ & 0.5627 \\
RRGPEQTQGNFGDQELIRQGTDYK & $\mathrm{Y}$ & 0.6277 \\
DPNFKD & $\mathrm{Y}$ & 2.878 \\
DAYKTFPPTEPKKDKKKKADETQALPQRQKKQQTVTLLPAADLDD & $\mathrm{Y}$ & 0.4968 \\
\hline
\end{tabular}

Table IIb. List of peptides that have the antigen properties and qualifies for the molecular docking process with human BCR/FAB Receptor.

\begin{tabular}{cc}
\hline Peptide & $\begin{array}{c}\text { Antigen Score } \\
\text { (Threshold = 0.4) }\end{array}$ \\
\hline HGKEDLKFPRGQGVPINTNSSPDDQIGYYRRATRRIRGGDGKMKDLS & 0.5773 \\
TTLPKGFYAGSRGGSQASSRSSSRSRNSSRNSTPGSSRGTSPARMAGNGGD & 0.5206 \\
RLNQLESKMSGKGQQQQGQTVTKKSAAEASKKPRQKRTATKA & 0.5627 \\
RRGPEQTQGNFGDQELIRQGTDYK & 0.6277 \\
DPNFKD & 2.878 \\
DAYKTFPPTEPKKDKKKKADETQALPQRQKKQQTVTLLPAADLDD & 0.4968 \\
\hline
\end{tabular}

Table III. Binding affinity or binding score that were obtained from each of the molecular docking processes. The binding score had been sorted from the highest score (most negative) to the lowest score (least negative).

\begin{tabular}{ccc}
\hline NO. & Peptide - BCR/FAB Interaction & Binding Score \\
\hline 1. & PEP1_BCR/FAB & $-726.5 \mathrm{~kJ}$ \\
2. & PEP2_BCR/FAB & $-701.2 \mathrm{~kJ}$ \\
3. & PEP3_BCR/FAB & $-537.5 \mathrm{~kJ}$ \\
4. & PEP4_BCR/FAB & $-502.0 \mathrm{~kJ}$ \\
5. & PEP5_BCR/FAB & $-391.9 \mathrm{~kJ}$ \\
6. & PEP6_BCR/FAB & $-607.0 \mathrm{~kJ}$ \\
\hline
\end{tabular}

not show any significant differences, and based on this result, the sequence that was chosen to be predicted in the B-cell epitope prediction is the sample from DKI Jakarta. From this B-Cell Epitope prediction that was conducted on the IEDB online webserver, a total of eleven predicted peptides were acquired (Figure 3). These peptides were generated into a list of peptides, which was inputted into a table based on their prediction score. To be included in the table, the peptide's prediction score needs to go above 0.5 (threshold). The results after the peptides had gone through the antigenicity test (Table IIa). To determine whether the peptides have antigen properties or not, the peptide's antigenicity score needs to pass or go above the threshold score (threshold score: 0.4). After going through the antigenicity test, only six peptides that have the antigen properties and qualifies to be docked along with the human BCR/FAB receptor. 


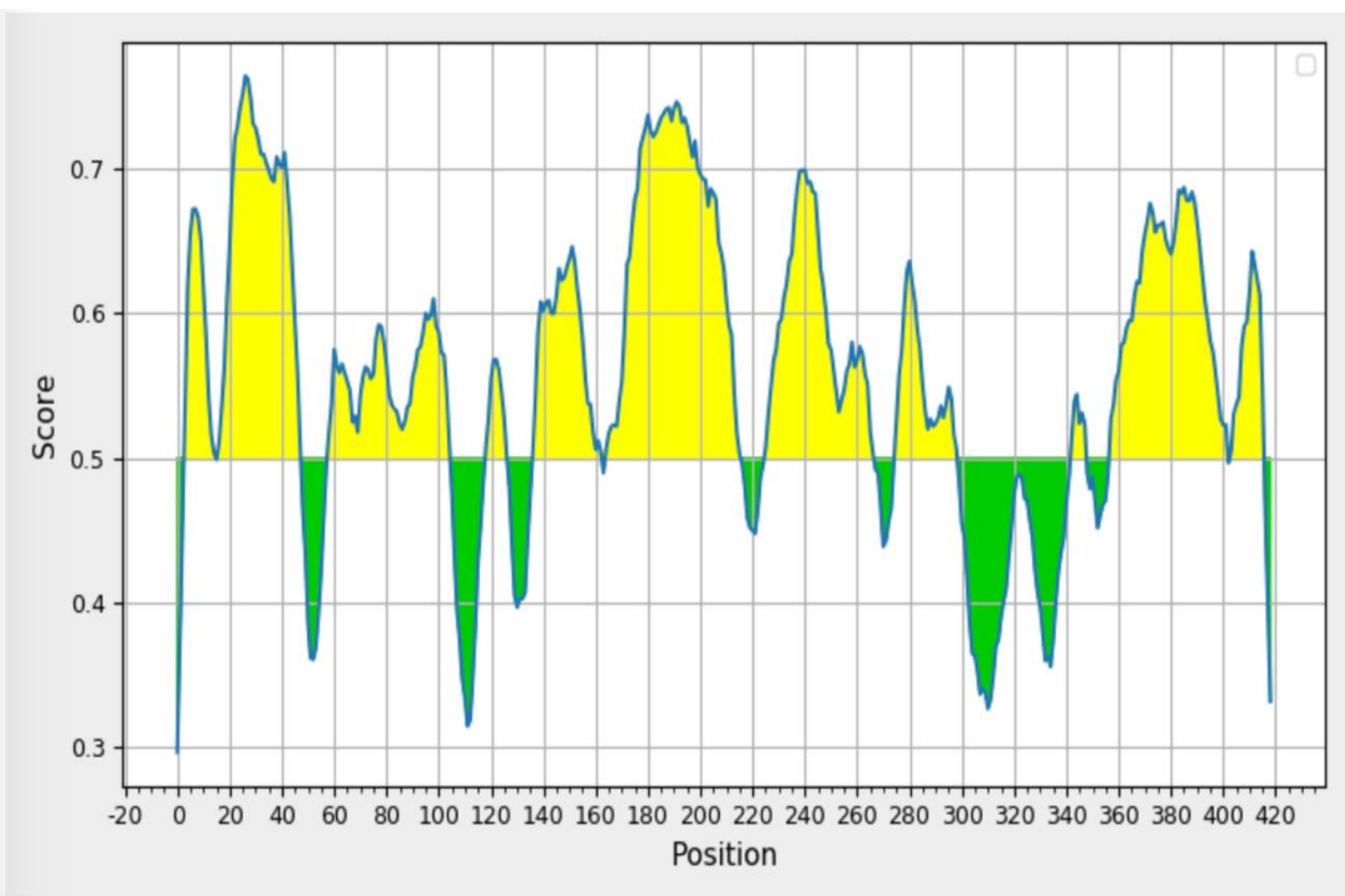

Figure 3. Prediction of B-cell epitopes from the amino acids of N protein of SARS-CoV-2. B-cell epitope prediction was performed using the IEDB online webserver. The region with yellow color indicates a positive prediction, meanwhile, the region with green color indicates a negative prediction of B-cell epitopes.

Meanwhile, the other five peptides will be excluded for the next process since they do not have any antigen features, which were needed to develop an epitope-based peptide vaccine (Table IIb).

But beforehand, those six peptides need to be modeled into 3D-Structures by using the PEPFOL3 Software. The main reason why it is needed to model the peptides into 3D-Structure is that the molecular docking process can only use 3DStructure (PDB file) as the input file and the expected result of the molecular docking process is a 3D-Structure of the peptides that bind with the receptor.

\section{Molecular docking and visualizations}

The first result shown from the molecular docking process is the visualization of the peptides that bind with the BCR/FAB fragment receptor (Figure 4). From the visualization can be seen that peptides bind perfectly with the BCR/FAB Fragment Receptor, however, this visualization needs to be further validated, and to validate the visualization, a binding affinity score/lowest binding score needs to be checked. To determine which peptides had the best binding affinity score, the most negative score in the results was chosen.

The binding scores of the six peptides after they are docked with the Human BCR/FAB receptors are shown (Table III). The first peptide (PEP1) after the docking process got the highest binding score (most negative) compared to the other six peptides with a score of -726.5. This means that the candidates for the Epitope-Based Vaccine were retrieved and based on the result (Table III) PEP1 (HGKEDLKFPRGQGVPINTNSSPDDQIGYYRRATR RIRGGDGKMKDLS) was proposes as the epitopebase peptide vaccine candidates to deal with the SARS-CoV-2 virus. BLASTp result with E value cut off of $10^{-3}$ shows that the PEP1 still has high conservation with nucleoprotein of SARS-CoV-2. The BLASTp result shows that PEP1 still elicits 


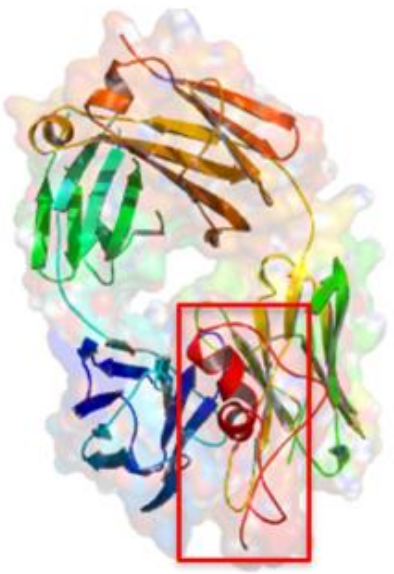

PEP1_BCR/FAB

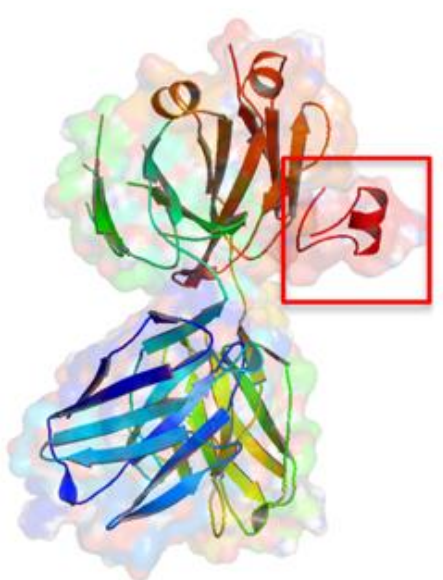

PEP4_BCR/FAB

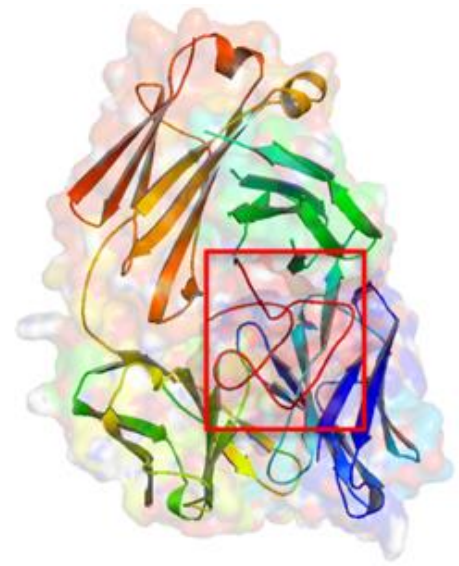

PEP2_BCR/FAB

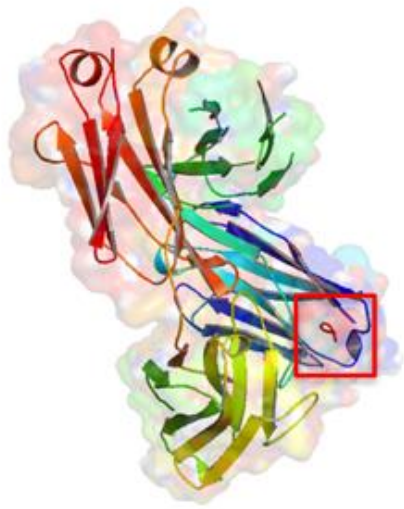

PEP5_BCR/FAB

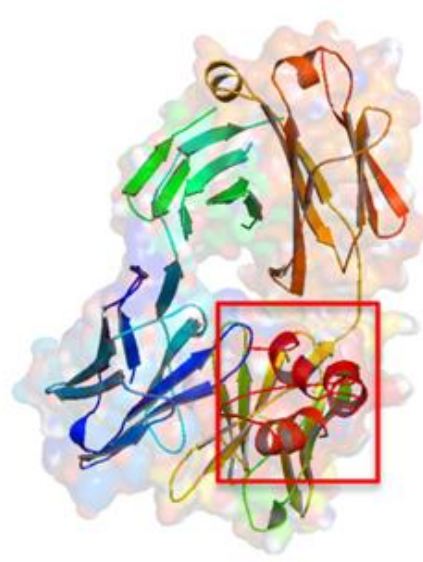

PEP3_BCR/FAB

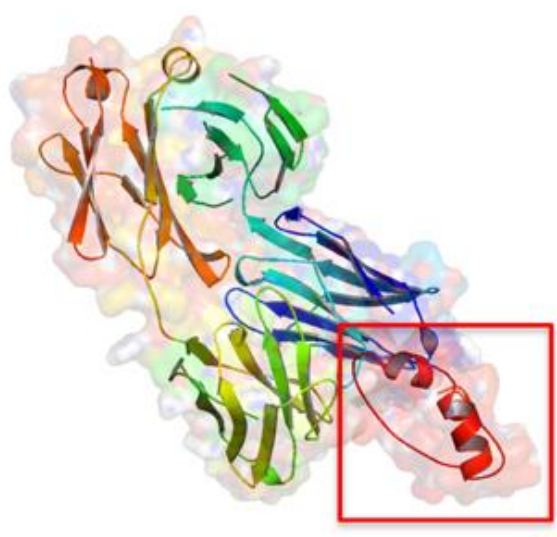

PEP6_BCR/FAB

Figure 4. Visualization results from the molecular docking process between each of the peptides with $\mathrm{BCR} / \mathrm{FAB}$ receptor in $3 \mathrm{D}$ structure visualization. It shows that each peptide binds perfectly with the BCR/FAB fragment receptor.

clearly defined properties of the SARS-CoV-2 nucleoprotein.

Then, more than $80 \%$ of the annotation SARS-CoV-2 mutations go to the spike protein, while the remaining was left mainly to the nucleoprotein (Troyano-Hernáez et al, 2021). Moreover, mutations in the SARS-CoV-2 protein only disrupt the drug-binding cavity, and there was no significant disruption in the epitope's elicitation (Azad, 2021). In this regard, PEP1 still has a higher probability to elicit an immune response as most of the mutation tendency was directed to the spike protein.

\section{CONCLUSION}

Based on the analysis that had been conducted and the results that had been retrieved, PEP1 was recommended as an epitope-based peptide vaccine candidate to deal with the SARSCoV-2 outbreaks. PEP1 was chosen because it has the highest level of immunogenicity, and it can be confirmed that it would not trigger an autoimmune. Also, it was shown that PEP1 is capable of forming BCR molecular complexes with the lowest binding energy for activation of transduction signal in the direct B-Cell immune response. In the future, to further validate the results of this study and to test 
the efficacy of this peptide vaccine candidate, molecular dynamics, in vitro, and in vivo testing could be done. Also, it is still possible to continue the study by developing a multi-epitope-based vaccine design in the future.

\section{ACKNOWLEDGEMENT}

The authors would like to thank the Department of Bioinformatics, School of Life Sciences, Center of Student Development Department (CSD), Research and Community Service Department (LPPM) of Indonesia International Institute for Life Sciences (i3L), and Generasi Biologi Indonesia Foundation for supporting this research. The authors would like to thank also the Deputi Bidang Penguatan Riset dan Pengembangan, Kementerian Riset dan Teknologi for providing Hibah Penelitian Dasar 2021 No B/112/E3/RA.00/2021. The authors also gratefully acknowledge the authors, the originating and submitting laboratories for their sequence and metadata shared through GISAID

\section{REFERENCES}

Ansori, A., Kharisma, V., Antonius, Y., Tacharina, M., and Rantam, F. 2020. Immunobioinformatics analysis and phylogenetic tree construction of severe acute respiratory syndrome coronavirus 2 (SARS-CoV-2) in Indonesia: Spike glycoprotein gene.Jurnal Teknologi Laboratorium, 9(1), 13-20.

Azad, G. K. (2021). The molecular assessment of SARS-CoV-2 Nucleocapsid Phosphoprotein variants among Indian isolates. Heliyon, $7(2)$, e06167. https://doi.org/10.1016/J.HELIYON.2021.E 06167

Benson, D. A., Karsch-Mizrachi, I., Lipman, D. J., Ostell, J., and Sayers, E. W. 2010. GenBank. Nucleic Acids Research, 38 (Database issue), D46-D51.

Bhattacharya, M., Sharma, A. R., Patra, P., Ghosh, P., Sharma, G., Patra, B. C., Lee, S. S., and Chakraborty, C. 2020. Development of epitope-based peptide vaccine against novel coronavirus 2019 (SARS-COV-2): Immunoinformatics approach. Journal of Medical Virology, 92(6), 618-631.

Brown, D. K., and Tastan Bishop, Ö. 2017. Role of structural bioinformatics in drug discovery by computational SNP analysis: Analyzing variation at the protein level. Global Heart, 12(2), 151-161.
Chen, H. Z., Tang, L. L., Yu, X. L., Zhou, J., Chang, Y. F., and $\mathrm{Wu}, \mathrm{X} .2020$. Bioinformatics analysis of epitope-based vaccine design against the novel SARS-CoV-2. Infectious Diseases of Poverty, 9, 88.

Dong, E., Du, H., and Gardner, L., 2020. An interactive web-based dashboard to track COVID-19 in real time. The Lancet. Infectious Diseases, 20(5), 533-534.

Doytchinova, I.A., and Flower, D.R. 2007. VaxiJen: A server for prediction of protective antigens, tumour antigens and subunit vaccines. $B M C$ Bioinformatics, 8, 4.

Hall, B. G. 2013. Building phylogenetic trees from molecular data with MEGA. Molecular Biology and Evolution, 30(5), 1229-1235.

Jespersen, M. C., Peters, B., Nielsen, M., and Marcatili, P. 2017. BepiPred-2.0: Improving sequence-based B-cell epitope prediction using conformational epitopes. Nucleic Acids Research, 45(W1), W24-W29.

Kao, D. J., and Hodges, R. S. 2009. Advantages of a synthetic peptide immunogen over a protein immunogen in the development of an antipilus vaccine for Pseudomonas aeruginosa.Chemical Biology \& Drug Design, 74(1), 33-42.

Kharisma, V. D., and Ansori, A. N. 2020. Construction of Epitope-Based Peptide Vaccine Against SARS-CoV-2: Immunoinformatics Study. Journal of Pure and Applied Microbiology, 14, 999-1005.

Kozakov, D., Hall, D. R., Xia, B., Porter, K. A., Padhorny, D., Yueh, C., Beglov, D., and Vajda, S. 2017. The ClusPro web server for proteinprotein docking. Nature Protocols, 12(2), 255-278.

Lesk, A. M., 2019. Bioinformatics. Retrieved November 14, 2020. https://www.britannica.com/science/bioin formatics.

Mcbride, R., Zyl, M. V., and Fielding, B. 2014. The coronavirus nucleocapsid is a multifunctional protein. Viruses, 6(8), 29913018.

Muttaqin, S. S., and Ansori, A. N. M. Candidate inhibitors of SARS-CoV-2 main protease with 3D structures similar to N3. Research Journal of Biotechnology, 15(11), 55-59.

Samrat, S. K., Tharappel, A. M., Li, Z., and Li, H. 2020. Prospect of SARS-CoV-2 spike protein: Potential role in vaccine and therapeutic development. Virus research, 288, 198141. 
Sanchez-Trincado, J. L., Gomez-Perosanz, M., and Reche, P. A. 2017. Fundamentals and Methods for T- and B-Cell Epitope Prediction. Journal of Immunology Research, 2017, 2680160.

Shen, Y., Maupetit, J., Derreumaux, P., and Tufféry, P. 2014. Improved PEP-FOLD approach for peptide and miniprotein structure prediction. Journal of Chemical Theory and Computation, 10(10), 4745-4758.

Shu, Y., and McCauley, J. 2017. GISAID: Global initiative on sharing all influenza data - from vision to reality. Euro Surveillance: Bulletin Europeen sur les maladies transmissibles = European Communicable Disease Bulletin, 22(13), 30494.

Steers, N. J., Currier, J. R., Jobe, O., Tovanabutra, S., Ratto-Kim, S., Marovich, M. A., Kim, J. H., Michael, N. L., Alving, C. R., and Rao, M. 2014. Designing the epitope flanking regions for optimal generation of CTL epitopes. Vaccine, 32(28), 3509-3516.
Thomas, J. A., and Gorelick, R. J. 2008. Nucleocapsid protein function in early infection processes. Virus Research, 134(1-2), 39-63.

Troyano-Hernáez, P., Reinosa, R., \& Holguín, Á. (2021). Evolution of sars-cov-2 envelope, membrane, nucleocapsid, and spike structural proteins from the beginning of the pandemic to september 2020: A global and regional approach by epidemiological week. Viruses, 13(2). https://doi.org/10.3390/v13020243

Tomar, N., and De, R. K. 2014. Immunoinformatics: A brief review. Methods in Molecular Biology, 1184, 23-55.

TopuzoĞullari, M., Acar, T., Pelİt Arayici, P., UÇar, B., UĞurel, E., Abamor, E. Ş., ArasoĞlu, T., Turgut-Balik, D., and Derman, S. 2020. An insight into the epitope-based peptide vaccine design strategy and studies against COVID-19. Turkish Journal of Biology, 44(3), 215-227.

WHO. 2020. Coronavirus. Retrieved September 16, 2021. https://www.who.int/healthtopics/coronavirus/coronavirus. 